\title{
Téoros
}

Revue de recherche en tourisme

\section{Parutions récentes}

Volume 20, numéro 1, printemps 2001

URI : https://id.erudit.org/iderudit/1071901ar

DOI : https://doi.org/10.7202/1071901ar

Aller au sommaire du numéro

\section{Éditeur(s)}

Université du Québec à Montréal

ISSN

0712-8657 (imprimé)

1923-2705 (numérique)

Découvrir la revue

Citer ce document

(2001). Parutions récentes. Téoros, 20(1), 82-83.

https://doi.org/10.7202/1071901ar

Ce document est protégé par la loi sur le droit d'auteur. L'utilisation des services d'Érudit (y compris la reproduction) est assujettie à sa politique d'utilisation que vous pouvez consulter en ligne.

https://apropos.erudit.org/fr/usagers/politique-dutilisation/
Cet article est diffusé et préservé par Érudit.

Érudit est un consortium interuniversitaire sans but lucratif composé de l’Université de Montréal, l'Université Laval et l'Université du Québec à Montréal. Il a pour mission la promotion et la valorisation de la recherche. https://www.erudit.org/fr/ 


\section{Parutions récentes}

\section{LA PREVISION-PROSPECTIVE EN GESTION Tourisme - Loisir - Culfure}

Jean Stafford et Bruno Sarrasin

\section{Collection Tourisme \\ Collection dirigée par Jean Stafford}

Les entreprises et les gouvernements ne peuvent plus ignorer la veille socioéconomique. En effet, pour survivre et se développer; toute organisation doit être en mesure de détecter les tendances dominantes dans son secteur d'activité. La collecte adéquate

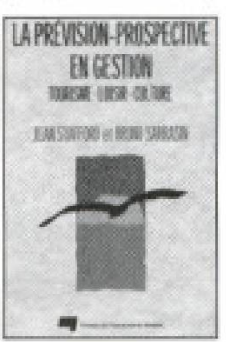
des données et la validité de ces données permettent de déceler des tendances qui conduisent à l'élaboration des stratégies appropriées, de politiques claires et à l'atteinte des succès espérés.

Les auteurs tentent d'appréhender le futur par le biais de méthodes rigoureuses de présentation et d'analyse des données, de manière à établir des passerelles entre le connu et l'inconnu, entre le possible et le souhaitable. Prévoir l'avenir exige rigueur et bon sens, mais aussi imagination et sensibilite a l'histoire en train de se faire. L'approche présentée ici repose sur quelques axiomes: pas de bonnes prévisions sans de bonnes données; par d'analyses prospectives valables sans de bonnes prévisions.

Jean Stafford détient un doctorat en sciences économiques, est spécialiste en tourisme et enseigne au département d'études urbaines et touristiques de l"Université du Québec à Montréal.

Bruno Sarrasin détient deux maîtrises, une en science politique et une autre en administration publique; il est chargé de cours en tourisme au département d'études urbaines et touristiques de I'Université du Québec à Montréal.

\section{DROIT DU TOURISME AU QUÉBEC}

Louis Jolin

\section{Collection Tourisme \\ Collection dirigée par Jean Stafford}

L'auteur présente les fondements de l'intervention législative et réglementaire dans le secteur du tourisme et analyse les prin= cipales règles de droit qui visent à protéger le consommateur dans le cadre d'une activité touristique. Une place centrale est accordée à la question de la responsabilité des fournisseurs de services au consommateur. L'auteur énonce et explique les lois protégeant le milieu d'accueil, notamment aux plans environnemental et culturel.

Dans cet ouvrage axé sur le droit en vigueur au Québec, l'auteur établit quelques comparaisons avec des lois d'autres provinces ou d'autres pays et traite des efforts entrepris à l'échelle inter-

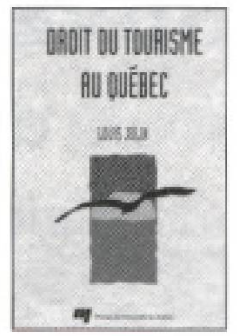

nationale pour régir les comportements touristiques dans leur ensemble.

Louis Jolin est professeur au département d'études urbaines et touristiques de l'École des sciences de la gestion de l'Université du Québec à Montréal. Diplồmé en droit de l'Université de Montréal et détenteur d'un doctorat en droit des affaires de l'Université Lyon 3, il enseigne le droit du tourisme depuis de nombreuses années et est actif au niveau international, notamment au sein du Bureau international de tourisme social dont il est l'un des vice-présidents et le président de la section des Amériques.

\section{L'INDUSTRIE TOURISTIQUE : strategie concurrentielle des entreprises}

\section{Vincent Sobourin}

\section{Collection Tourisme}

Collection dirigée par Jean Stafford, Marc Laplante et Sylvie Gagnon

Cet ouvrage décrit les différents concepts liés à la stratégie commerciale et à la planification stratégique de l'industrie touristique dans un environnement concurrentiel.

L'auteur y traite d'abord de l'élaboration et du choix des stratégies en fonction du produit et de l"environnement dans une perspective visant à réconcilier les notions de marché et de concurrence avec celles de métiers, de compétences, de savoir-faire et de culture organisationnelle. Il montre comment

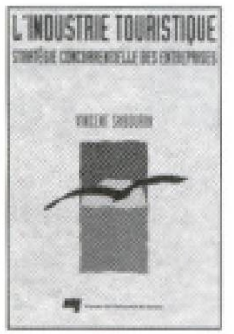
les entreprises performantes dans l'industrie touristique innovent en combinant des activités de façon originale, afin d'offrir de meilleurs produits, de rejoindre de nouvelles clientèles ou encore d'étendre leurs activités.

L'auteur présente également les stratégies concurrentielles développées par les leaders du marché comme les chaînes hôttelières Marriott, Holiday Inn et Four Seasons, les compagnies aériennes telles British Airways, Southwest et Singapour Airline, et les entreprises de divertissement comme Virgin Atlantic, Carnival, Walt Disney et le Cirque du Soleil.

Vincent Sabourin, Ph.D., est professeur de stratégie de gestion à l'École des sciences de la gestion de l'Université du Québec à Montréal et enseigne au programme de la maîtrise en planification et gestion du tourisme. Il a effectué des interventions comme expert dans le domaine du tourisme pour des réseaux d'hébergement, des projets de développement immobilier et des associations sectorielles. Il anime des séminaires à travers le Canada et à l'étranger. 


\section{Parutions récentes}

\section{LESPACE TOURISTIQUE}

Sous la direction de Normand Cazelais. Roger Nadeau et Gérard Beaudet

\section{Collection Tourisme \\ Collection dirigée par Jean Stafford, Marc Laplante et Sylvie Gagnon}

Dans cet ouvrage, le premier du genre publié au Québec, les auteurs présentent, à l'ensemble des enseignants, chercheurs, consultants, planificateurs et gestionnaires, une dimension spécifique du phếnomène

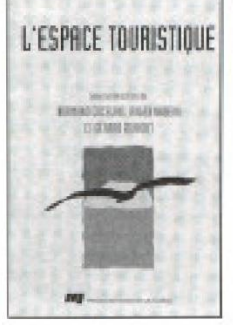
touristique : le territoire.

L'expansion de la vocation touristique de ce territoire á été considérable tout au long du XX' siècle et le Québec n'y a pas échappé en s'inscrivant dans la mouvance nord-américaine dès le milieu du XIX ${ }^{2}$ siècle. Les textes réunis ici confirment cette appartenance et rendent compte tout à la fois des modalités de l'émergence de l'espace touristique, de son organisation et des particularités de son aménagement au gré des tendances du développement régional et de l'évolution des sensibilités, des attentes et des préoccupations des visiteurs et des visités.

Par des exemples puisés au Québec et ailleurs, les auteurs proposent un survol des caractéristiques qui déterminent l'attractivité et la vocation d'un site. Ils abordent les différents types d'espaces touristiques et se penchent sur les relations entre visiteurs et visités. Ils dépeignent l'ampleur du défi que doivent relever les concepteurs, les promoteurs, les aménagistes et les gestionnaires de territoires et d'equipements touristiques. Ils proposent finalement une lecture de la production et de lá structuration de l'espace touristique, ainsi que des processus du façonnement de l'offre.

Normand Cazelais est géographe, spécialiste en environnement à Hydro-Québec et chroniqueur au quotidien Le Devoir.

Roger Nadeau est professeur au département de géographie de l'Université de Sherbrooke.

Gérard Beaudet est professeur à l'Institut d'urbanisme et chercheur à la Chaire en paysage et environnement de la Faculté de l'aménagement de l'Université de Montréal.

\section{L'ARCHITECTURE DE SAINT-ROCH : guide de promenade}

\author{
Lucie K. Morisset et Luc Noppen
}

\section{Les Publications du Québec, (2000)}

Saint-Roch, ce petit bourg de Québec baigné par la Saint-Charles, s'est métamorphosé peu à peu en capitale industrielle, puis en haut lieu de l'américanité avec ses places cinématographiques, ses tramways et ses grands magasins. Délaissé pendant plusieurs annees, Saint-Roch est redevenu ce milieu débordant de vitalité où foisonnent de nouvelles constructions et des rếnovations qui témoignent bien de l'engouement actuel pour ce paysage urbain.

L'architecture de Saint-Roch : guide de promenade permet de mesurer pleinement l'évolution architecturale de ce quartier de Québec et, à travers elle, sa riche histoire. Les auteurs nous convient à une visite captivante en 46 tableaux, concis mais fouillés, articulés autour de huit thèmes offrant un tour d'horizon complet. On y trouve au-delà de 240 illustrations qui ajoutent au plaisir de la découverte. Enfin, une carte placée à la fin du

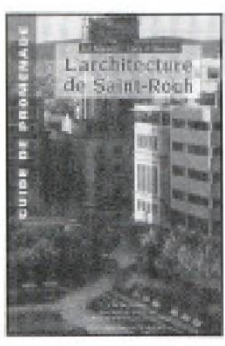
volume permet de situer plus de 150 bâtiments d'intérêt présentés au fil des pages. Une invitation à découvrir ces architectures remarquables lors d'une balade dans les rues de Saint-Roch!

Luc Noppen et Lucie K. Morisset sont professeurs à l'Université du Québec à Montréal. Historiens de l'architecture, ils sont tous deux chercheurs au Centre d'études interdisciplinaires sur les lettres, les arts et les traditions (CÉLAT/UQAM). Ils ont signé plusieurs livres, articles et rapports de recherche sur l'architecture et sur l'histoire du Québec et ont obtenu plusieurs prix prestigieux. 